\title{
Magnetic marker localisation in breast cancer surgery
}

Jan Žatecký, Otakar Kubala, Petr Jelínek, Milan Lerch, Peter Ihnát, Matúš Peteja, Radim Brát

Department of Surgical Studies, Faculty of Medicine, University of Ostrava, Ostrava, Czech Republic

Submitted: 26 April 2019; Accepted: 12 July 2019

Online publication: 14 March 2020

Arch Med Sci 2023; 19 (1): 122-127

DOI: https://doi.org/10.5114/aoms.2020.93673

Copyright $\odot 2020$ Termedia \& Banach

\section{Abstract}

Since mammographic screening programmes were initiated, the spectrum of breast cancer has changed in terms of impalpable tumours, thus causing the development of new localisation methods, including magnetic markers. We offer herein an up-to-date review focused on two magnetic markers (Magseed, MaMaLoc) currently used in breast cancer surgery for the localisation of breast tumours or pathological axillary nodes. Magnetic marker localisation presents a safe and reliable method for breast tumour marking. Four currently available prospective studies demonstrate that the Magseed system has a negative margin rate and a successful localisation rate, both of which are comparable to standard marking systems used in breast cancer surgery. The main benefits of magnetic markers are that they require no radiation safety measures, and they offer the possibility of longer deployment times, thus simplifying surgery scheduling. The most important drawbacks are cost of the system, depth limitation and need for frequent probe recalibration.

Key words: breast cancer surgery, magnetic marker, impalpable breast tumour, Magseed, MaMaLoc.

\section{Introduction}

Breast cancer is the most common worldwide malignancy in women, and its incidence is increasing due to the success of mammography screening programmes, which enable the detection of small and often non-palpable tumours [1]. At present, $25-35 \%$ of diagnosed breast cancer tumours are non-palpable [2]. As a result, techniques using markers for precise localisation of the tumour have been developed, with markers being introduced by a radiologist into the centre or to the periphery of the tumour, thus simplifying its detection during surgery.

Targeted axillary dissection (TAD) presents another important option in breast cancer surgery, where the employment of reliable markers is of paramount importance. Targeted axillary dissection consists of a sentinel lymph node biopsy (SLNB) and the excision of the pathological lymph node, which has been marked before neoadjuvant therapy. If metastasis is found in the sentinel lymph node or in a marked pathological node, axillary dissection (AD) levels I and II are performed. Targeted axillary dissection seems to be more accurate (by virtue of the false-negativity rate) than SLNB only, especially in women after neoadjuvant therapy with initially node-positive axillary status [3], although it is dependent on the reliability of the used marker.

\author{
Corresponding author: \\ Jan Žatecký \\ 233 Slezská St \\ Neplachovice, 74774 \\ Czech Republic \\ E-mail: \\ janzatecky.jz@gmail.com
}


Table I. Summary of magnetic localisation methods

\begin{tabular}{|lccccc|}
\hline Method & Size & $\begin{array}{c}\text { Number of } \\
\text { prospective cohorts }\end{array}$ & $\begin{array}{c}\text { Magnetic resonance } \\
\text { imaging compatibility }\end{array}$ & Detectable up to & $\begin{array}{c}\text { Commercially } \\
\text { available }\end{array}$ \\
\hline Megseed & $1 \times 5 \mathrm{~mm}$ & 4 & $\begin{array}{c}\text { Yes (bloom effect } \\
\text { up to } 4-6 \mathrm{~cm})\end{array}$ & $30 \mathrm{~mm}$ & Yes \\
\hline MaMaLoc & $1.5 \times 3.5 \mathrm{~mm}$ & 1 & No & $35 \mathrm{~mm}$ & No \\
\hline
\end{tabular}

Apart from standard markers in breast cancer surgery (such as wire localisation or metal clips), magnetic markers present a promising new option for breast tumour localisation. A literature search revealed that published data on the topic are highly insufficient, and so the aim of the present paper is to offer an up-to-date review focused on magnetic marker localisation in breast cancer surgery.

\section{Non-magnetic markers}

Many techniques are used nowadays for localising non-palpable breast tumours. Wire-guided localisation (WGL) is a widely used method, and it was first reported in 1965 [4]. The most commonly published disadvantages of WGL are patient discomfort, wire migration or transection, limitations to surgical incisions because of wire placement, vaso-vagal episodes and complications regarding surgery scheduling (WGL must be performed on the same day as surgery) [4-7].

Implanted tissue marker clips are markers without a specific detection system, and therefore preoperative localisation by WGL or a specimen radiograph (after excision of the lesion for confirmation that the clip is included) is necessary. Clip migration and problematic clip localisation present the main difficulties of the technique [8].

Carbon marking is a cheap marking technique that creates a tattoo in place of an injection, but it can imitate malignancy as a result of foreign-body giant-cell reaction $[7,9,10]$.

Radioactive seed localisation (RSL) using iodine-125 (125l) seeds was first described in 1999 by Dauway et al. [11]. Since then, authors of several studies have demonstrated the non-inferiority of RSL compared to WGL [12-14]. The main advantage of RSL is that the seed can be put in place many days or even weeks before surgery, which allows for much easier surgery scheduling. The main RSL disadvantage rests in radiation safety regulations.

In the last few years, several new and non-radioactive non-wire localisation methods have appeared. SAVI SCOUT uses infrared light and radar technology, whereby the marker $(12 \times 4 \mathrm{~mm}$ with two $4 \mathrm{~mm}$-long antennas) is detected by a handpiece and console system [5, 6]. Available data suggest that SAVI SCOUT is comparable to WGL in terms of both the negative margin and the re-

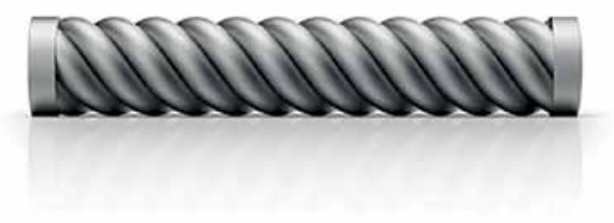

Figure 1. Magseed - $1 \times 5 \mathrm{~mm}$ stainless steel magnetic localisation seed (with permission of Sysmex CZ Ltd)

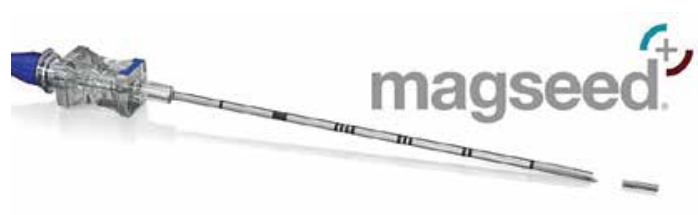

Figure 2. Sterile introducer and Magseed (with permission of Sysmex CZ Ltd)

excision rates. However, its main limitations are cost, nickel content relating to the risk of allergy reaction, device failure by interaction with electrocautery and high directionality of the system $[5,6]$.

Radiofrequency identification tags (RFIDs) are based on radio wave transmission and contain a microprocessor in which information can be stored [7]. It has a history of usage as an identification device, e.g. for pets, but the first clinical data about intraoperative use are very promising, with one advantage over other localisation technologies - the probe can detect distance from the tag $[15,16]$.

\section{Magnetic markers}

At present, there are two markers that use magnetic susceptibility to localise tumours in breast cancer surgery - Magseed and MaMaLoc (Table I).

Magseed (Endomagnetics, Inc.) was approved by the FDA for breast lesion localisation in 2016 [6]. The method utilises $1 \times 5 \mathrm{~mm}$ stainless steel magnetic seeds (Figure 1) implanted by an $18 \mathrm{G}$ sterile introducer (Figure 2) under mammography or ultrasound guidance (Figure 3). After Magseed implantation, the marker position cannot be changed, which is similar to other nonwire markers [4]. During surgery, the seed is de- 
tected by a handheld magnetometer, SentiMag (Endomagnetics, Inc.) (Figure 4) at a distance up to $30 \mathrm{~mm}$ away $[17,18]$. If the Magseed is placed deeper than $30 \mathrm{~mm}$, it may not be detectable, but using palpation with a probe, which means tissue compression, deeper Magseeds are possible to detect. The Magseed may be implanted safely up to 30 days before surgery, but ongoing clinical trials are currently investigating a longer implantation

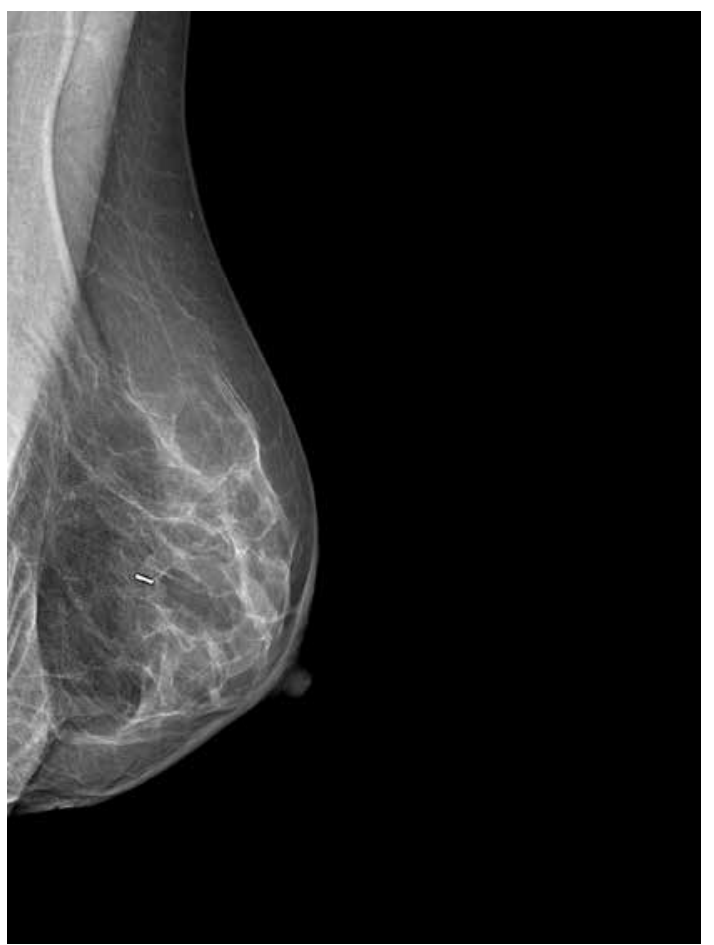

Figure 3. Mammogram with Magseed implementation at the site of the breast tumour (University Hospital Ostrava)

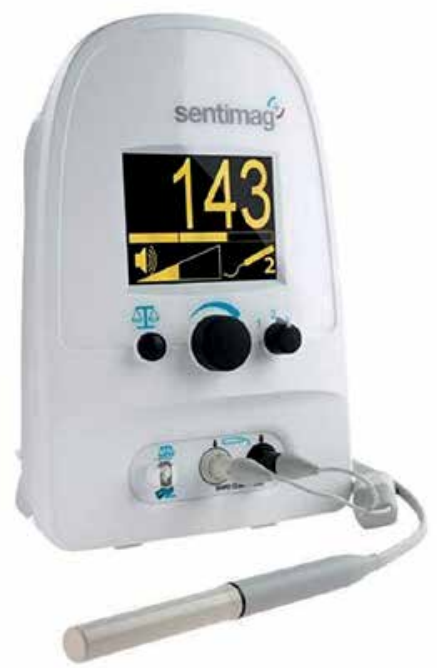

Figure 4. Handheld Sentimag magnetometer (with permission of Sysmex CZ Ltd)

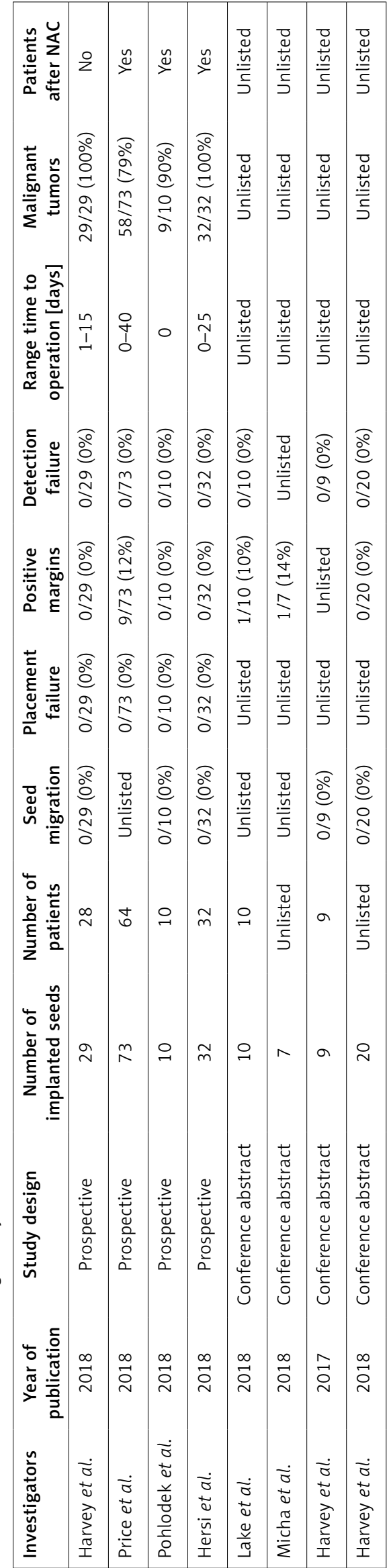


period, which could be crucial in breast tumours or positive axillary lymph node localisation in patients after neoadjuvant therapy (NAC), which usually lasts 3-4 months. The main limitation of the Magseed system is noted when magnetic resonance imaging (MRI) is part of the treatment protocol, e.g. restaging after NAC. Although the Magseed is MRI-compatible, it has a bloom effect of up to 4-6 cm [5, 7], which renders radiological evaluation of the tumour in breast tissue unfeasible.

To date, there are only four prospective studies and four conference abstracts offering clinical data regarding Magseed magnetic markers (Table II). The highest number of implanted seeds ( 73 seeds in 64 patients) was published by Price et al. [18], but data regarding Magseed migration are unfortunately lacking in this study. The other three prospective studies, working with 10, 29 and 32 implanted Magseeds, reported $0 \%$ seed migration. Moreover, the authors of all four studies reported $100 \%$ successful placement and detection of the seed in patients with various breast volumes [17-20]. Harvey et al. reported a correlation between breast size and the ease of detecting the Magseed, in that the detection time was shorter in smaller breasts, and the initial count on the SentiMag probe was higher [17]. Dorsal tumour localisation in large breasts may cause problems in detecting the Magseed, but the palpation of the seed may help with initial localisation. Unsuccessful detection of the Magseed has not been described in the literature to date.

Positive/negative resection margins present an extremely important criterion of localisation method reliability in breast cancer surgery. Negative margins are defined as clear margins without tumour cells or at a distance 1 or $2 \mathrm{~mm}$ away from the breast tumour, according to various authors [21, 22]. Markers are usually implanted into the centre of the tumour. Marker implantation and detection accuracy contribute to achievement of negative margins. Three prospective studies presented $0 \%$ positive margins in all patients with Magseed localisation [17, 19, 20]. The main limitation of these outcomes is a low number of included patients (10, 28 and 32 patients). Price et al., for instance, reported a positive resection margin in $12 \%$ of 64 patients with 73 seeds [18]. In comparison with other localisation methods, WGL has a negative margin rate of $70-88 \%$ [9], RSL 73.5-96.7\% [5], clip markers 90-92\% [23] and SAVI SCOUT 85.1-92.6\% [5]. According to these first results, Magseed localisation seems to be comparable with other breast localisation markers in terms of oncosurgical radicality. A prospective comparative study between Magseed and other markers is currently lacking.
The Magseed system can be used to localise multiple lesions in the same breast. The limitation is the proximity of lesions under $2 \mathrm{~cm}$, which may result in the inability of the magnetometer to separate signals [18]. However, it is questionable whether it is actually necessary to separate signals emanating from two close lesions during breast-conserving surgery, because for negative margin achievement, surgeons need to excise a sufficient amount of the surrounding tissue.

The interference of the Magseed signal with electrocautery or paramagnetic surgical instruments is another very important limitation of magnetic marker localisation techniques $[4,6,9$, 24]. Non-conductive instruments (i.e. polymer or carbon fibre) need to be used while scanning with a Sentimag probe [5], but it is not necessary to use non-conductive instruments during the entire surgical procedure. In case of interference with magnetic instruments, the Sentimag probe needs to be recalibrated [6]. The frequent need for recalibration is one of the biggest drawbacks of the Sentimag system.

The localisation of pathological axillary nodes for targeted axillary dissection (TAD) by means of a Magseed system presents another very promising option in breast cancer surgery [17]. According to NCCN guidelines, TAD is a possible option in staging axillary status after neoadjuvant treatment in patients with initially metastatic axillary nodes. Using only SLNB, the false-negativity rate stands at over $10 \%$ in this group of patients, which is unacceptable, especially when one recognises that, in comparison, TAD has a false-negativity rate of $2 \%$ [3]. Marking of the pathological node in axilla is dependent on a safe and reliable marker and its localisation system. According to the literature, various localisation methods are used - RSL [3], clip markers [25] or carbon marking [26], each with its own advantages and disadvantages as discussed above. Magseed properties such as no marker migration and intuitive detection $[17,19]$ could be crucial in this area, but no clinical study has been published on this topic in the field of breast cancer surgery, although two prospective open-label studies are currently ongoing at the M.D. Anderson Cancer Center (NCT03038152) [27], (NCT03796559) [28].

Two prospective studies (Pohlodek et al. 2018, Hersi et al. 2018) have investigated the simultaneous use of Magseed and SLNB with the magnetic tracer Sienna (Endomagnetics, Inc.) [19, 20], which is a solution containing superparamagnetic iron oxide nanoparticles that are injected into the breast pre-operatively and accumulate in the sentinel lymph node through lymphatics, before detection by a Sentimag probe [19]. The au- 


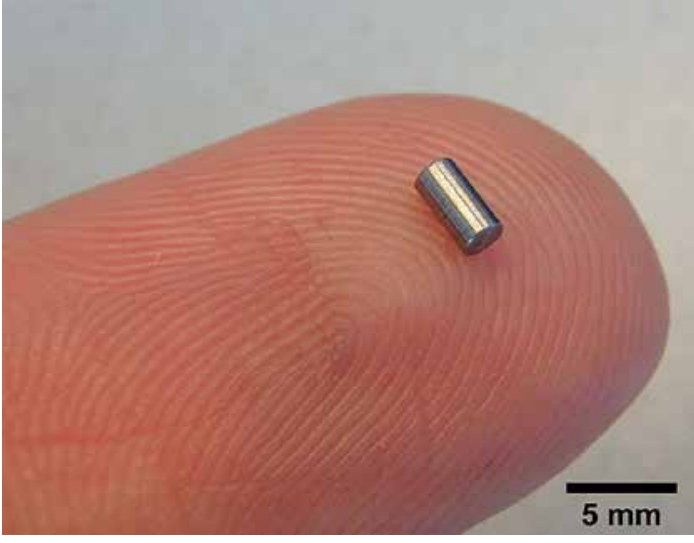

Figure 5. MaMaLoc marker - $1.5 \times 3.5 \mathrm{~mm}$ magnetic localisation marker (with permission of Sirius Medical Systems B.V.)

thors found no interference between these two magnetic methods - Magseed for breast lesion localisation or Sienna for SLNB - when using the same Sentimag probe for detection [19, 20], and so they concluded that a combination of Magseed and Sienna should reduce the use of radioactive methods in breast cancer surgery $[19,20]$. The successful separate usage of Sienna for SLNB has been reported in prospective studies since 2014 [29-31]; moreover, in 2016, the first metaanalysis was done by Karakatsanis et al., indicating that the effectiveness of the Sienna system is comparable to standard techniques using a radioactive tracer [32].

The main drawback of Magseed is the cost of the system, in that the price of the Sentimag probe and each implanted magnetic seed is much higher in comparison to wire-guided localisation [17, 20]. Harvey et al. proposed that a full cost analysis (considering factors such as faster scheduling in the operating theatre, reduced restrictions due to radiation safety policy and patient satisfaction) could balance out the higher Magseed price [17].

The MaMaLoc (magnetic marker localisation) system, which is another magnetic marker used for breast tumour localisation, was developed at The Netherlands Cancer Institute [33]. The only prospective single-centre study regarding the MaMaLoc system (Figure 5), for which 15 female patients with non-palpable breast cancer tumours for surgical therapy without NAC were recruited, was published in 2017 [33]. All MaMaLoc markers were placed successfully 5-30 days before surgery, and a radioactive ${ }^{125}$ s seed reference marker was also placed in each patient. MaMaLoc migration ranged from $0 \mathrm{~mm}$ to $0.5 \mathrm{~mm}$, which is clinically irrelevant, because it does not change the site of operation. MaMaLoc detection was provided by a Sentimag probe (Endomagnetics, Inc.). The identification rate for the marker during breast-conserving surgery was $100 \%$, and all tumours were excised. The study does not provide evidence about positive/negative resection margins of excised tumours, but these criteria are scheduled for assessment in an ongoing MaMaLoc-2 trial (NTR6767) [34].

MaMaLoc is not compatible with MRI. Because the Sentimag probe is used, there is a recommendation as in Magseed to use non-conductive tools during the surgery whilst detecting magnetic markers. MaMaLoc technology is not currently commercially available, but The Netherlands Cancer Institute has created a spin-off company to spread the MaMaLoc system worldwide. Magseed is commercially available in many countries in Europe, North America, Asia, Africa and Australia.

\section{Conclusions}

Magnetic marker localisation is a safe and reliable method for breast tumour marking. There are four prospective studies available, each of which demonstrates that the Magseed system has a negative margin rate and a successful localisation rate comparable to standard marking systems used in breast cancer surgery. The main benefits of magnetic markers are that they require no radiation safety measures, and they offer the possibility of longer deployment times, thus simplifying surgery scheduling. The most important drawbacks are the cost of the system, depth limitation and need for frequent probe recalibration.

\section{Acknowledgments}

This article was supported by the Student Grant Competition of the University of Ostrava.

\section{Conflict of interest}

The authors declare no conflict of interest.

\section{References}

1. Wyld L, Markopoulos C, Leidenius M, Senkus-Konefka E (eds.). Breast Cancer Management for Surgeons: A European Multidisciplinary Textbook. Springer International Publishing 2018.

2. Patel SN, Mango VL, Jadeja P, et al. Reflector-guided breast tumor localization versus wire localization for lumpectomies: a comparison of surgical outcomes. Clin Imaging 2018; 47: 14-7.

3. Caudle AS, Yang WT, Krishnamurthy S, et al. Improved axillary evaluation following neoadjuvant therapy for patients with node-positive breast cancer using selective evaluation of clipped nodes: implementation of targeted axillary dissection. J Clin Oncol 2016; 34: 1072-8.

4. Spalluto LB, DeBenedectis CM, Morrow MS, Lourenco AP. Advances in breast localization techniques: an opportunity to improve quality of care and patient satisfaction. Semin Roentgenol 2018; 53: 270-9. 
5. Cheang E, Ha R, Thornton CM, Mango VL. Innovations in image-guided preoperative breast lesion localization. Br J Radiol 2018; 91: 20170740.

6. Jeffries DO, Dossett LA, Jorns JM. Localization for breast surgery: the next generation. Arch Pathol Lab Med 2017; 141: 1324-9.

7. Hayes MK. Update on preoperative breast localization. Radiol Clin North Am 2017; 55: 591-603.

8. Dash N, Chafin SH, Johnson RR, Contractor FM. Usefulness of tissue marker clips in patients undergoing neoadjuvant chemotherapy for breast cancer. Am J Roentgenol 1999; 173: 911-7.

9. Green M, Vidya R. Techniques used to localize occult breast lesions: an update. Clin Breast Cancer 2018; 18: e281-3.

10. Ruiz-Delgado ML, López-Ruiz JA, Sáiz-López A. Abnormal mammography and sonography associated with foreign-body giant-cell reaction after stereotactic vacuum-assisted breast biopsy with carbon marking. Acta Radiol 2008; 49: 1112-8.

11. Dauway EL, Saunders R, Friedland J. Innovative diagnostics for breast cancer: new frontiers for the new millennium using radioactive seed localization. Surgical forum: $85^{\text {th }}$ annual American college of surgeons clinic congress. Vol. 50. Chicago, IL: American College of Surgeons; 1999.

12. Sharek D, Zuley ML, Zhang JY, Soran A, Ahrendt GM, Ganott MA. Radioactive seed localization versus wire localization for lumpectomies: a comparison of outcomes. AJR Am J Roentgenol 2015; 204: 872-7.

13. Langhans L, Tvedskov TF, Klausen TL, et al. Radioactive seed localization or wire-guided localization of nonpalpable invasive and in situ breast cancer: a randomized, multicenter, open-label trial. Ann Surg 2017; 266: 29-35.

14. Sung JS, King V, Thornton CM, et al. Safety and efficacy of radioactive seed localization with I-125 prior to lumpectomy and/or excisional biopsy. Eur J Radiol 2013; 82: $1453-7$.

15. Dauphine C, Reicher JJ, Reicher MA, Gondusky C, Khalkhali I, Kim M. A prospective clinical study to evaluate the safety and performance of wireless localization of nonpalpable breast lesions using radiofrequency identification technology. AJR Am J Roentgenol 2015; 204: W720-3.

16. DiNome ML, Kusske AM, Attai DJ, Fischer CP, Hoyt AC. Microchipping the breast: an effective new technology for localizing non-palpable breast lesions for surgery. Breast Cancer Res Treat 2019; 175: 165-70.

17. Harvey JR, Lim Y, Murphy J, et al. Safety and feasibility of breast lesion localization using magnetic seeds (Magseed): a multi-centre, open-label cohort study. Breast Cancer Res Treat 2018; 169: 531-6.

18. Price ER, Khoury AL, Esserman LJ, Joe BN, Alvarado MD. Initial clinical experience with an inducible magnetic seed system for preoperative breast lesion localization. AJR Am J Roentgenol 2018; 210: 913-7.

19. Pohlodek K, Foltín M, Mečiarová I, Ondriaš F. Simultaneous use of magnetic method in localization of impalpable breast cancer and sentinel lymph nodes detection: initial experience. Nanomedicine 2018; 13: 3075-81.

20. Hersi AF, Eriksson S, Ramos J, Abdsaleh S, Wärnberg F, Karakatsanis A. A combined, totally magnetic technique with a magnetic marker for non-palpable tumour localization and superparamagnetic iron oxide nanoparticles for sentinel lymph node detection in breast cancer surgery. Eur J Surg Oncol 2019; 45: 544-9.
21. Pilewskie M, Morrow M. Margins in breast cancer: how much is enough? Cancer 2018; 124: 1335-41.

22. Houssami N, Macaskill P, Marinovich ML, et al. Metaanalysis of the impact of surgical margins on local recurrence in women with early-stage invasive breast cancer treated with breast-conserving therapy. Eur J Cancer 2010; 46: 3219-32.

23. Corsi F, Sorrentino L, Bossi D, Sartani A, Foschi D. Preoperative localization and surgical margins in conservative breast surgery. Int J Surg Oncol 2013; 2013: 793819.

24. Chacko SM, Marshall HN. Implementation of preoperative magnetic seed localization for breast and axillary lesions: an alternative to wires and radioactive seeds. J Radiol Nurs 2018; 37: 154-7.

25. Coufal O, Zapletal O, Gabrielová L, Fabian P, Schneiderová M. Targeted axillary dissection and sentinel lymph node biopsy in breast cancer patients after neoadjuvant chemotherapy - a retrospective study. Rozhl Chir 2018; 97: 551-7.

26. Lee J, Jung JH, Kim WW, et al. 5-year oncological outcomes of targeted axillary sampling in pT1-2N1 breast cancer. Asian J Surg 2019; 42: 681-7.

27. Study the Use of Magseed and Sentimag to Localize Axillary Lymph Nodes - Full Text View - ClinicalTrials. gov [Internet]. Available at: https://clinicaltrials.gov/ ct2/show/NCT03038152 (Accessed: 27.02.2019).

28. Magseed Enabled Long-Term Localization of Axillary Lymph Nodes - Full Text View - ClinicalTrials.gov [Internet]. Available at: https://clinicaltrials.gov/ct2/show/ NCT03796559 (Accessed: 4.02.2019).

29. Thill M, Kurylcio A, Welter R, et al. The Central-European SentiMag study: sentinel lymph node biopsy with superparamagnetic iron oxide (SPIO) vs. radioisotope. Breast 2014; 23: 175-9.

30. Ghilli M, Carretta E, Di Filippo F, et al. The superparamagnetic iron oxide tracer: a valid alternative in sentinel node biopsy for breast cancer treatment. Eur J Cancer Care (Engl) 2017; 26. doi: 10.1111/ecc.12385.

31. Douek M, Klaase J, Monypenny I, et al.; SentiMAG Trialists Group. Sentinel node biopsy using a magnetic tracer versus standard technique: the SentiMAG Multicentre Trial. Ann Surg Oncol 2014; 21: 1237-45.

32. Karakatsanis A, Christiansen PM, Fischer L, et al. The Nordic SentiMag trial: a comparison of super paramagnetic iron oxide (SPIO) nanoparticles versus Tc(99) and patent blue in the detection of sentinel node (SN) in patients with breast cancer and a meta-analysis of earlier studies. Breast Cancer Res Treat 2016; 157: 281-94.

33. Schermers B, van der Hage JA, Loo CE, et al. Feasibility of magnetic marker localisation for non-palpable breast cancer. Breast 2017; 33: 50-6.

34. MaMaLoc: a study on the use of a magnetic marker for tumor localization in breast cancer surgery. Trialregister. $\mathrm{nl}$ [Internet]. Available at: https://www.trialregister.nl/ trial/6553 (Accessed: 27.02.2019). 